\title{
Terapia médico-nutricional en el paciente con quilotórax: reporte de caso
}

\author{
Medical-Nutrition therapy in the patient with chylothorax: \\ A case report \\ Terapia médico-nutricional na paciente com quilotórax: \\ relato de caso
}

\author{
Ricardo Rendón-Rodríguez ${ }^{1 *}$, Iván Armando Osuna-Padilla², \\ Karen Viané Orozco-Hernández ${ }^{3}$, Francisco Javier Roberto Enríquez-Reyes ${ }^{3}$
}

Recibido: 22 de marzo de 2019. Aceptado para publicación: 8 de julio de 2019.

Publicado en línea: 24 de julio de 2019

https://doi.org/10.35454/rncm.v3n1.014

\begin{abstract}
Resumen
Introducción: el quilotórax es una condición caracterizada por derrame pleural secundaria a lesión o bloqueo del conducto torácico. El volumen del quilo drenado es influenciado por los lípidos presentes en la alimentación (nutrición enteral o vía oral), por lo que el reposo del tracto gastrointestinal es una práctica común en esta población. En la actualidad no existe consenso acerca de la vía de alimentación asociada a un menor gasto de quilo y a una recuperación más acelerada.

Caso clínico: se presenta el caso clínico de un paciente con diagnóstico de quilotórax secundario a bloqueo del conducto torácico por linfoadenopatía tratado con nutrición mixta (parenteral y vía oral). Se reporta la evolución del gasto quiloso así como los cambios en el perfil metabólico y de electrolitos durante los primeros siete días de abordaje nutricional.

Conclusiones: la administración de la terapia nutricional mixta (oral y NP) permitió la disminución del gasto quiloso. Se observaron cambios en la composición y aspecto del mismo. Se propone un algoritmo para la intervención de estos pacientes en los centros hospitalarios.
\end{abstract}

Palabras clave: quilotórax, estado nutricional, nutrición enteral, nutrición parenteral, lípidos.

\section{Summary}

Introduction: chylothorax is a condition characterized by pleural effusion secondary to injury or blockage of the thoracic duct. The volume of drained chyle is influenced by the lipids present in the diet (enteral or oral nutrition), so rest of the gastrointestinal tract is a common practice in this population. At present, there is no consensus about the feeding path associated with a lower output of chylous and a more accelerated recovery.

Clinical case: the clinical case of a patient with a diagnosis of chylothorax secondary to blockage of the thoracic duct by lymphadenopathy treated with mixed nutrition (parenteral and oral) is presented. We report the evolution of chylous output as well as the changes in the metabolic and electrolyte profile during the first seven days of the nutritional approach.

Conclusions: the administration of mixed nutritional therapy (oral and PN) allowed the reduction of chylous output. Changes in the composition and appearance of the chylous were observed. An algorithm is proposed for the intervention of these patients in hospital centers.

Keywords: Chylothorax; Nutritional status; Enteral nutrition; Parenteral nutrition, Lipids.

\section{Resumo}

Introdução: o quilotórax é uma condição caracterizada por derrame pleural secundário a lesão ou bloqueio do ducto torácico. O volume do quilo drenado é influenciado pelos lipídios presentes na dieta (nutrição enteral ou oral), de modo que o repouso do trato gastrointestinal é uma prática comum nessa população. Atualmente, não há consenso sobre a via de alimentação associada a um menor gasto com quilo e a uma recuperação mais acelerada.

Caso clínico: é apresentado o caso clínico de um paciente com diagnóstico de quilotórax secundário ao bloqueio do ducto torácico por linfadenopatia tratado com nutrição mista (nutrição parenteral e oral). É relatada a evolução do gasto quiloso, bem como as mudanças no perfil metabólico e eletrolítico durante os primeiros sete dias da abordagem nutricional.

Conclusões: a administração de terapia nutricional mista (oral e NP) permitiu a diminuição do gasto quiloso. Observaramse mudanças na sua composição e aparência. Um algoritmo é proposto para a intervenção nestes pacientes em centros hospitalares.

Palavras-chave: quilotórax, estado nutricional, nutrição enteral, nutrição parenteral, lipídios.
1. Departamento de Nutrición. Hospital General de Cholula (HGCH). Puebla, México.

2. Centro de Investigación en Enfermedades Infecciosas (CIENI), Instituto Nacional de Enfermedades Respiratorias (INER). Ciudad de México.

\section{Servicio de Cirugía. Hospital General de Cholula (HGCH). Puebla, México.}

*Correspondencia: Ricardo Rendón-Rodríguez ricardo_rendon@outlook.com 


\section{INTRODUCCIÓN}

El quilotórax es una condición clínica que se genera como consecuencia de una lesión o bloqueo del conducto torácico, en la cual existe la acumulación de quilo (líquido linfático procedente del tracto gastrointestinal, especialmente del intestino delgado) en la cavidad pleural, el cual está compuesto en forma principal por lípidos, albúmina y linfocitos (Tabla 1). La excreción persistente de quilo conduce al deterioro del estado nutricional y al desarrollo de alteraciones en la respuesta inmunológica, como consecuencia de las pérdidas de proteínas y otros micronutrientes en conjunto con el impacto metabólico de la patología de base ${ }^{(1)}$.

Tabla 1. Características del quilotórax

\begin{tabular}{|l|l|}
\hline Aspectos físicos & $\begin{array}{l}\text { Aspecto lechoso } \\
\text { pH alcalino: 7,4-7,8 } \\
\text { Estéril }\end{array}$ \\
\hline $\begin{array}{l}\text { Aspectos } \\
\text { bioquímicos }\end{array}$ & $\begin{array}{l}\text { Proteínas totales: } 20-60 \mathrm{~g} / \mathrm{L} \\
\text { Albúmina: } 1,2-4,1 \mathrm{~g} / \mathrm{dL} \\
\text { Grasa total: } 0,4-6 \mathrm{~g} / \mathrm{dL}\end{array}$ \\
& $\begin{array}{l}\text { Triglicéridos: }>110 \mathrm{mg} / \mathrm{dL} \\
\text { Colesterol: } 65-220 \mathrm{mg} / \mathrm{dL} \\
\text { Relación triglicéridos líquido } \\
\text { pleural/suero }>1 \\
\text { Relación colesterol líquido } \\
\text { pleural/suero }<1 \\
\text { Linfocitos (principalmente células T): } \\
400-7.000 / \mathrm{mm}^{3}\end{array}$ \\
\hline
\end{tabular}

Adaptado de Sirvent Ochando y col. ${ }^{(1)}$

El diagnóstico de quilotórax se establece cuando la concentración de triglicéridos y colesterol en el aspirado pleural es superior a los $110 \mathrm{mg} / \mathrm{dL}$ y menor a $200 \mathrm{mg} / \mathrm{dL}$, respectivamente, o bien cuando el cociente colesterol/triglicéridos es $<1$, aunque en $15 \%$ de los casos diagnosticados los triglicéridos se encuentran por debajo de $110 \mathrm{mg} / \mathrm{dL}$, documentán- dose concentraciones menores de $50 \mathrm{mg} / \mathrm{dL}$ en $3 \%$ de los pacientes ${ }^{(2)}$.

Los criterios de Light permiten clasificar el derrame pleural de acuerdo con la composición de los líquidos serosos en el trasudado o exudado; la ausencia de criterios corresponde a un derrame trasudado, siendo categorizado como derrame exudado aquellos casos que cumplen uno o más criterios (Tabla 2). En la mayoría de los casos de quilotórax (75\%), los criterios de Light describen las características del exudado ${ }^{(2)}$. Otros criterios diferenciales para el tipo de derrame pleural han mostrado una menor capacidad diagnóstica ${ }^{(3,4)}$.

El tratamiento del quilotórax se basa principalmente en medidas conservadoras, como la instauración del drenaje por sonda endopleural, la modificación de los macronutrientes de la dieta y el uso de medicamentos para la reducción del volumen de las secreciones (somatostatina o su análogo octreótide). El tratamiento quirúrgico (decorticación, anastomosis linfovenosa, pleurodesis mecánica, pleurectomía parietal o reparación del conducto torácico), ya sea por toracoscopia o toracotomía, está indicado en casos donde existe una pérdida mayor a $1.500 \mathrm{~mL} /$ día durante más de 5 días o si el drenaje continúa después de dos semanas de tratamiento conservador ${ }^{(5)}$.

En México la frecuencia de esta condición clínica ronda $0,03 \%$. La etiología es diversa, reportes indican que $50 \%$ de los casos ocurren por malignidad mediastínica, en donde los linfomas representan $75 \%$ de los casos de origen tumoral, mientras que $20 \%$ - $25 \%$ de los casos son por complicaciones quirúrgicas. Considerando la baja incidencia, no existen estudios prospectivos que permitan identificar el abordaje (conservador o quirúrgico) más adecuado. El tiempo de instauración del tratamiento conservador es incierto, pero en promedio los casos reportan la resolución en 15 días. Algunos autores consideran terapia fallida la ausencia de disminución del drenaje tras 7 - 10 días desde su inicio ${ }^{(6-8)}$.

Tabla 2. Criterios de Light

\begin{tabular}{|l|c|c|l|l|}
\hline & $\begin{array}{c}\text { P: LDH } \\
\text { pleural/suero }\end{array}$ & $\begin{array}{c}\text { P: Proteínas } \\
\text { pleural/suero }\end{array}$ & LDH pleural & \multicolumn{1}{|c|}{ Entidades clínicas } \\
\hline Trasudado & $<0,6$ & $<0,5$ & $\begin{array}{l}>2 / 3 \text { partes del límite superior } \\
\text { normal de LDH plasmática. }\end{array}$ & $\begin{array}{l}\text { Hipoalbuminemia, cirrosis hepática, } \\
\text { síndrome nefrótico, hipotiroidismo. }\end{array}$ \\
\hline Exudado & $>0,6$ & $>0,5$ & $\begin{array}{l}>2 / 3 \text { partes del límite superior } \\
\text { normal de LDH plasmática. }\end{array}$ & $\begin{array}{l}\text { Tuberculosis, malignidades, enfermedades } \\
\text { autoinmunes, infarto pulmonar. }\end{array}$ \\
\hline
\end{tabular}

LDH: Lactado deshidrogenasa. P: Proporción 


\section{ABORDAJE NUTRICIONAL}

El conducto torácico transporta en promedio 2,4 L de quilo al día, el cual está compuesto por $70 \%$ de los lípidos ingeridos en la dieta, es por ello que el tratamiento nutricional es un pilar fundamental en su tratamiento.

La terapia nutricional tiene por objetivo:

a. Prevenir el deterioro del estado nutricional del paciente, reponiendo los nutrimentos y fluidos perdidos en las secreciones.

b. Mejorar la evolución clínica del paciente, disminuyendo los costos y los días de estancia hospitalaria ${ }^{(2)}$.

El abordaje nutricional consiste en una alimentación baja en lípidos, en especial de triglicéridos de cadena larga (TCL), ya que estimulan las secreciones gástricas que se conducen a través de la linfa, priorizando el aporte de triglicéridos de cadena media (TCM), los cuales son absorbidos en el enterocito y transportados directamente a la circulación portal, sin necesidad de la secreción de enzimas pancreáticas ${ }^{(9)}$. Este tipo de lípidos no ha mostrado beneficios en la disminución del volumen del quilo secretado; sin embargo, su uso permite cubrir las necesidades energéticas del paciente ${ }^{(10)}$.

Los TCM, formados por un glicerol y tres ácidos grasos de cadena media (AGCM), se encuentran principalmente en el aceite de coco o de palma. Es importante mencionar que el consumo de AGCM no previene la deficiencia de ácidos grasos esenciales (AGE), los cuales son de cadena larga (ACGL) y de naturaleza poliinsaturada. Las principales fuentes alimentarias de AGE son los aceites vegetales y aceites de pescado ${ }^{(11,12)}$.

La instauración de algoritmos de abordaje nutricional en este tipo de patología permite la toma de decisiones en función del estado nutricional, mejorando con ello el pronóstico clínico del paciente, en la Figura 1 proponemos un algoritmo para el proceso de atención nutricional basado en los hallazgos de la evaluación del estado nutricional.

La prescripción de la terapia nutricional de manera personalizada a cada paciente resulta esencial para prevenir la desnutrición o deficiencia de AGE, las cuales suelen presentarse tras la prescripción de dietas bajas en TCL durante más de 7 días. Una estrategia para evitar esta deficiencia es el aporte diario de $25 \mathrm{~g}$ de aceite de soya, girasol o maíz, lo cual permite cubrir $3 \%$ del valor energético total (VET) en forma de ácido linoleico en una dieta promedio de 2.000 calorías $^{(13-16)}$.

De forma histórica, la selección de la vía de alimentación ha sido tema de debate, ya que se ha reportado un incremento en la estimulación del drenaje linfático inclusive al realizar restricción de lípidos en dieta enteral o vía oral, por lo que algunos autores contraindican dichas vías. Sin embargo, es importante considerar que la ausencia de estímulo intestinal ocasiona mayores complicaciones relacionadas con la atrofia de las vellosidades intestinales, condición que se asocia a translocación bacteriana, sepsis, falla orgánica múltiple y muerte, es por ello que se sugiere mantener la permeabilidad intestinal utilizando dosis tróficas de $\mathrm{NE}$, evaluando diariamente el impacto en el volumen de quilo secretado ${ }^{(17,18)}$.

El reposo digestivo a través del uso de NPT ha demostrado más casos de éxito, con resolución de hasta $84 \%$ de los casos, sin embargo, otros autores han reportado casos de éxito tras el uso de NE o dieta vía oral $\left.{ }^{(1,5,6,8,19,20}\right)$. $\mathrm{Al}$ no existir un consenso respecto a la vía de alimentación a utilizar, la toma de decisión deberá considerar el juicio clínico del profesional de la salud y la discusión con el equipo multidisciplinario ${ }^{(20,21)}$.

La inclusión de lípidos en la NP no representa mayor complicación ya que estos se entregan directamente a la circulación sanguínea. Se debe tener especial cuidado cuando se prescriban fármacos que disminuyen las secreciones gástricas, como los análogos de la Somatostatina (octreotide), ya que disminuyen el flujo sanguíneo intestinal y pueden ocasionar intolerancia gastrointestinal ${ }^{(20,22)}$.

No existen estudios que evalúen el gasto energético en esta condición clínica, por lo que se sugiere la medición del gasto energético con calorimetría indirecta. En ausencia de esta tecnología, se sugiere realizar la estimación de energía utilizando la ecuación Harris Benedict o $25 \mathrm{kcal} / \mathrm{kg}$ de peso/día ${ }^{(1,8,23)}$.

La medición del balance nitrogenado y la cuantificación de las pérdidas de proteínas en el líquido pleural drenado en 24 horas permiten determinar los requerimientos de proteínas, sugiriéndose la prescripción de 1,5 a $1,7 \mathrm{~g} / \mathrm{kg}$ de peso / día ${ }^{(1,8,23)}$.

\section{DESCRIPCIÓN DEL CASO}

Paciente masculino de 60 años de edad, con diabetes mellitus de tipo 2 de 20 años de diagnóstico, hipertensión arterial con 2 años de diagnóstico sin tratamiento, carcinoma epidermoide de pulmón y datos clínicos de falla hepática aguda. Refiere iniciar su padecimiento dos meses previos con presencia de astenia, adinamia, taquipnea y disnea a medianos esfuerzos, con presencia de tos sin expectoración, afebril. A la exploración física 


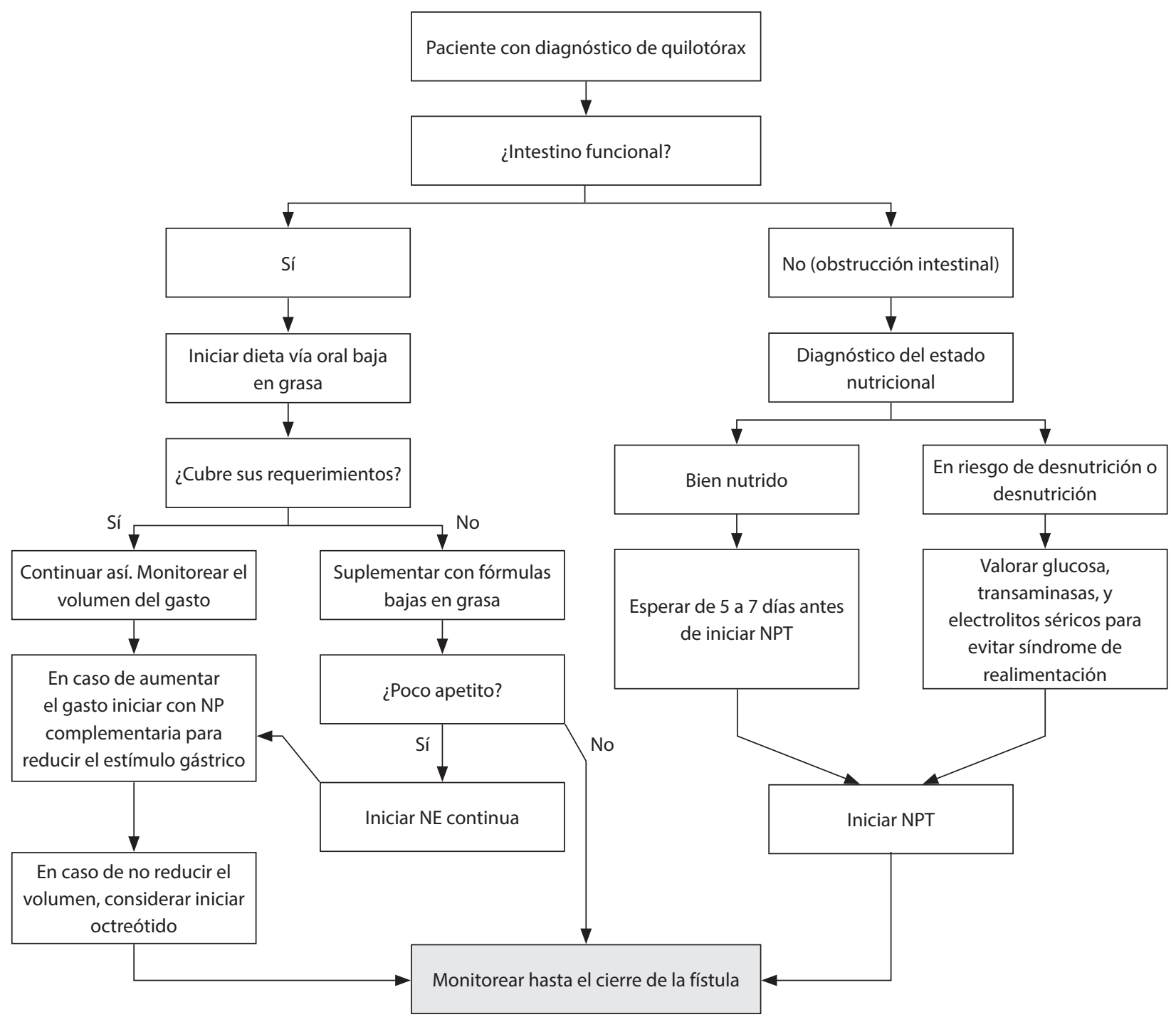

Figura 1. Algoritmo: propuesta de intervención.

se observa distensión abdominal, a la palpación se identifica edema abdominal y de extremidades inferiores.

$\mathrm{Al}$ ingreso se reporta una saturación de $84 \%$, FR 23 rpm, FC 103 lpm, tensión arterial y temperatura normales, en equilibrio ácido base según gasometría venosa ( $\mathrm{pH} 7,43, \mathrm{PaCO}_{2} 31,4 \mathrm{mmHg}, \mathrm{PaO}_{2} 46,8 \mathrm{mmHg}$, HCO3 20,6 mmol/L, EB -2,2 mmol/L, Lactato 1,4 $\mathrm{mmol} / \mathrm{L})$. Se procede con apoyo suplementario de oxígeno con puntas nasales a $3 \mathrm{~L} / \mathrm{min}$ manteniendo saturaciones de $89 \%$. Neurológicamente normal, 15 puntos en la escala de Glasgow sin posturas ni reflejos anormales.
La dificultad respiratoria progresa a pequeños esfuerzos. Se realiza radiografía de tórax la cual evidencia derrame pleural en hemitórax derecho (Figura 2) por lo que se procede a realizar toracocentesis, obteniéndose líquido color blanquecino con aspecto lechoso, al análisis arrojó los siguientes resultados: $\mathrm{pH} 8$, glucosa $283 \mathrm{mg} / \mathrm{dL}$, triglicéridos $293 \mathrm{mg} / \mathrm{dL}$, colesterol $45 \mathrm{mg} / \mathrm{dL}$, proteínas totales $1 \mathrm{~g} / \mathrm{L}$ y un cociente coleste$\mathrm{rol} /$ triglicéridos $<1(0,15)$ siendo el resultado positivo para diagnóstico de quilotórax secundario a bloqueo del conducto torácico por linfoadenopatía, el derrame pleural es considerado exudado por su etiología onco- 
lógica (quilotórax maligno). Se realiza toracotomía del hemitórax derecho para retirar cadena ganglionar, la cual se envía a estudio de patología, reportando infiltración neoplásica de carcinoma epidermoide de pulmón moderadamente diferenciado. Se decide colocar sonda endopleural al nivel de quinto espacio intercostal línea media axilar, realizándose drenaje en el día con un volumen total de $1.630 \mathrm{cc}^{(7)}$.

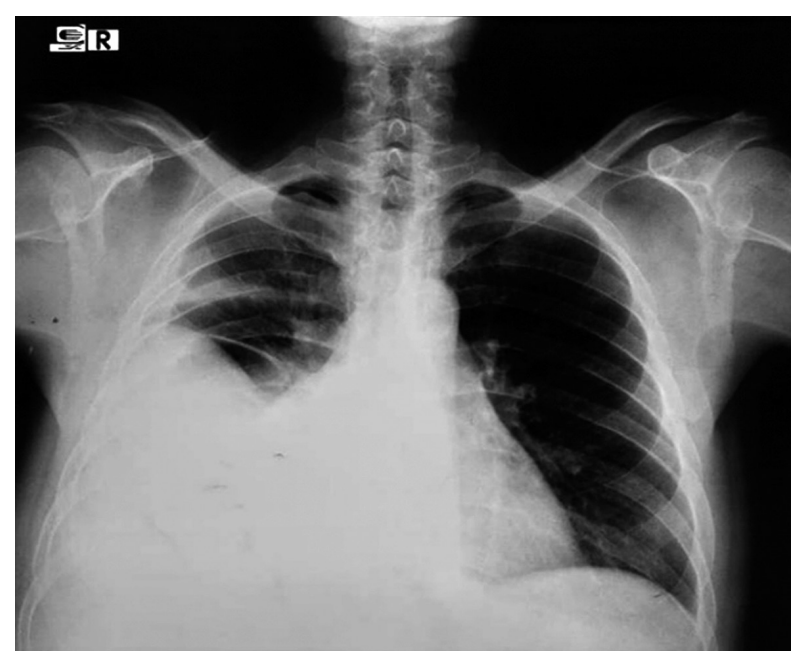

Figura 2. Radiografía de tórax que muestra derrame pleural derecho de $65 \%$ aproximadamente.

Se opta por tratamiento conservador con nutrición especializada y drenaje torácico. Se solicita interconsulta al Departamento de Nutrición, obteniéndose los siguientes datos en la valoración nutricional (Tabla 3). Las necesidades energéticas se estimaron utilizando la ecuación de Harris-Benedict y factor de 1,3 y un requerimiento proteico de $1,5 \mathrm{~g} / \mathrm{kg}$ de peso ideal / día (contabilizando las pérdidas de nitrógeno urinario, del drenaje pleural y las pérdidas insensibles) con un requerimiento de $97,5 \mathrm{~g} /$ día $^{(1)}$.

Considerando el estado nutricional del paciente $y$ su perfil electrolítico, se decide preservar la funcionalidad del tracto gastrointestinal a través de la vía oral con modificaciones dietéticas (verduras hervidas, frutas y carnes magras más $30 \mathrm{~g}$ de aceite vegetal para evitar la deficiencia de AGE, aportando $970 \mathrm{kcal}$ al día, prescribiéndose nutrición parenteral complementaria (NPC) con un aporte de $1.000 \mathrm{kcal}(400 \mathrm{~mL}$ de solución glucosada a $50 \%$ y $1.000 \mathrm{~mL}$ de aminoácidos al $8 \%$ +1 ampolla de multivitaminas), aportando un total de $1.969 \mathrm{kcal}$ y $100 \mathrm{~g}$ de proteínas (requerimiento $97,5 \mathrm{~g}$ / día) con un aporte de 97,5\% de sus requerimientos, con el objetivo de disminuir el gasto quiloso y reponer las pérdidas de macro y micronutrientes ${ }^{(24)}$.

El primer día de inicio de la terapia nutricional el paciente consumió $60 \%$ de la dieta prescrita, la cual consistía en pechuga de pollo, arroz cocido, chayote hervido y aceite de soya, mostrando buena tolerancia gastrointestinal. La dieta en los días 2, 3 y 4 estuvo compuesta por carne molida de res, pasta, ensalada de verduras y aceite de soya, esto para proporcionar variedad a la dieta, reportando un incremento en el apetito, logrando consumir el $80 \%$ de sus requerimientos energéticos.

La glucemia sérica del primer día fue de $305 \mathrm{mg} / \mathrm{dL}$, para el quinto día aumentó a $700 \mathrm{mg}$ / dL por lo cual se decide suspender la NP y se inicia esquema de insulina, una vez estable el paciente se reinicia la NP. En este día el drenaje pleural total disminuye de $920 \mathrm{cc}$ a $50 \mathrm{cc}$. En los días 6 y 7 el drenaje pleural disminuye a $20 \mathrm{cc}$, considerándose exitosa la terapia conservadora (Tabla 4).

Tabla 3. Evaluación del Estado Nutricional (ABCD)

\begin{tabular}{|c|c|c|c|}
\hline Antropometría & Bioquímicos & Clínicos & Dietéticos \\
\hline $\begin{array}{l}\text { Peso actual: } 70 \mathrm{~kg} \\
\text { Peso habitual: } 80 \mathrm{~kg} \text { (hace } 6 \text { meses) } \\
\text { Pérdida de peso: } 10 \mathrm{~kg} \text { ( } 8 \% \text {, pérdida } \\
\text { significativa). } \\
\text { Peso teórico: } 65 \mathrm{~kg} \\
\text { Pliegue cutáneo tricipital: } 10 \mathrm{~mm} \\
\text { (percentil < 5) } \\
\text { Área muscular del brazo corregida: } \\
18,9 \mathrm{~cm}^{2} \text { (percentil <5) }\end{array}$ & $\begin{array}{l}\text { Glucosa } 305 \mathrm{mg} / \mathrm{dL} \\
\text { Albúmina } 2,4 \mathrm{~g} / \mathrm{dL} \\
\text { Proteínas totales } 6,2 \mathrm{~g} / \mathrm{dL} \\
\text { LDH: } 227 \mathrm{UI} / \mathrm{L} \\
\text { GGT: } 1069 \mathrm{UI} / \mathrm{L} \\
\text { ALT: } 179 \mathrm{UI} / \mathrm{L} \\
\text { AST: } 139 \mathrm{UI} / \mathrm{L}\end{array}$ & $\begin{array}{l}\text { Examen físico: resequedad } \\
\text { cutánea, signos de } \\
\text { deshidratación leve y depleción } \\
\text { severa de masa muscular y de } \\
\text { tejido adiposo. } \\
\text { Medicamentos: } \\
\text { Metoclopramida, Senosidos } \\
\text { AB, Ketorolaco, Meropenem, } \\
\text { Vancomicina, Omeprazol, } \\
\text { Gluconato de calcio, Insulina } \\
\text { Glargina }\end{array}$ & $\begin{array}{l}\text { Análisis de recordatorio } \\
\text { de } 24 \text { horas: consumo de } \\
875 \text { kcal, } 43 \text { g de proteínas, } \\
\text { representando } 44,4 \% \text { de los } \\
\text { requerimientos de energía y } \\
\text { el } 44,1 \% \text { de proteína. }\end{array}$ \\
\hline
\end{tabular}


Tabla 4. Análisis del líquido pleural

\begin{tabular}{|c|c|c|c|c|c|c|c|}
\hline Días & Día 1 & Día 2 & Día 3 & Día 4 & Día 5 & Día 6 & Día 7 \\
\hline $\begin{array}{l}\text { Volumen total } \\
\text { drenado }(\mathrm{mL})\end{array}$ & 1630 & 1290 & 1110 & 920 & 50 & 20 & 20 \\
\hline \multicolumn{8}{|l|}{ Citológico } \\
\hline Aspecto & Lechoso & Hemorrágico & Turbio & Turbio & Turbio & Turbio & Turbio \\
\hline Color & Amarillo & Rojo & Amarillo & Blanco & Blanco & Blanco & Blanco \\
\hline $\mathrm{pH}$ & 8 & 9 & 7 & 6,5 & 7,5 & 8 & 8 \\
\hline \multicolumn{8}{|l|}{ Citoquímico } \\
\hline Colesterol (mg/dL) & 45 & 50 & 45 & 45 & 45 & 45 & 45 \\
\hline Triglicéridos (mg/dL) & 293 & 189 & 83 & 193 & 181 & 171 & 125 \\
\hline $\begin{array}{l}\text { Cociente: Colesterol/ } \\
\text { Triglicéridos }\end{array}$ & 0,15 & 0,26 & 0,54 & 0,23 & 0,24 & 0,26 & 0,36 \\
\hline
\end{tabular}

En el día 8 se suspende la NP quedando solo con dieta vía oral, sus signos vitales mostraban taquicardia, taquipnea y fiebre. Los niveles de albúmina sérica se mantuvieron disminuidos durante su estancia a pesar de recibir sus necesidades energéticas y proteicas, pues dicho parámetro bioquímico en este caso no refleja el estado nutricional del paciente sino la respuesta inflamatoria, jugando un papel como marcador de inflamación de fase aguda negativa, lo anterior confirmando el proceso inflamatorio con un valor de proteína $\mathrm{C}$ reactiva (PCR) de $90 \mathrm{mg} / \mathrm{L}$. Sin embargo, antes de su evaluación nutricional semanal, el paciente entra en paro cardiorrespiratorio y fallece.

\section{DISCUSIÓN}

En la actualidad no existe consenso acerca del abordaje nutricional del paciente con quilotórax. En el caso aquí presentado se decidió tratar nutricionalmente al paciente según los estándares actuales los cuales se basan, en general, en los casos descritos en la literatura científica, es decir, la instauración de la alimentación vía oral en conjunto con la nutrición parenteral. Este tratamiento condujo a la disminución del flujo quiloso sin necesidad de instaurar terapia farmacológica (Figura 3).

La disminución del volumen drenado como consecuencia de la terapia nutricional se considera un abordaje exitoso. En una serie de 17 casos de quilo- tórax reportado por un hospital español, 15 de estos casos fueron tratados con modificaciones dietéticas, realizando restricción de lípidos, los cuales eran predominantemente TCM, similar a la que recibió nuestro paciente. En su publicación, los autores no mencionan si los pacientes recibieron algún aporte de nutrientes vía parenteral, concluyendo que los quilotórax de bajo gasto pueden ser tratados de esta manera mientras que los de alto gasto requieren técnicas invasivas ${ }^{(20)}$. En el caso presentado por Merchán y col ${ }^{(5)}$ el paciente con un quilotórax neoplásico recibió solo modificaciones dietéticas vía oral debido a que no se disponía de NPT. Su evolución fue desfavorable necesitando intervención quirúrgica. Por el contrario, en el caso de Machado y $\mathrm{col}^{(8)}$ con un quilotórax traumático tratado con modificaciones dietéticas vía oral, la evolución fue favorable. En el caso presentado por Carrillo-Esper R y col ${ }^{(6)}$ un paciente con quilotórax traumático recibió únicamente NPT, teniendo una evolución favorable. En dos casos de quilotórax reportados de distinta etiología (posquirúrgico y neoplásico), la NPT y el uso de análogos de la somatostatina tuvieron efecto favorable en la evolución del quilotórax ${ }^{(1,20)}$. La decisión de tratar nuestro paciente con NP y dietoterapia se basó en la necesidad de mantener la integridad intestinal tal y como lo proponen Machado y col ${ }^{(8)}$, obteniendo una disminución del volumen total drenado, de $1.630 \mathrm{cc}$ a $20 \mathrm{cc}$ entre el día 1 y el día 7 , sin necesidad de cirugía ni prescripción de análogos de la somatostatina. Por lo tanto, según 


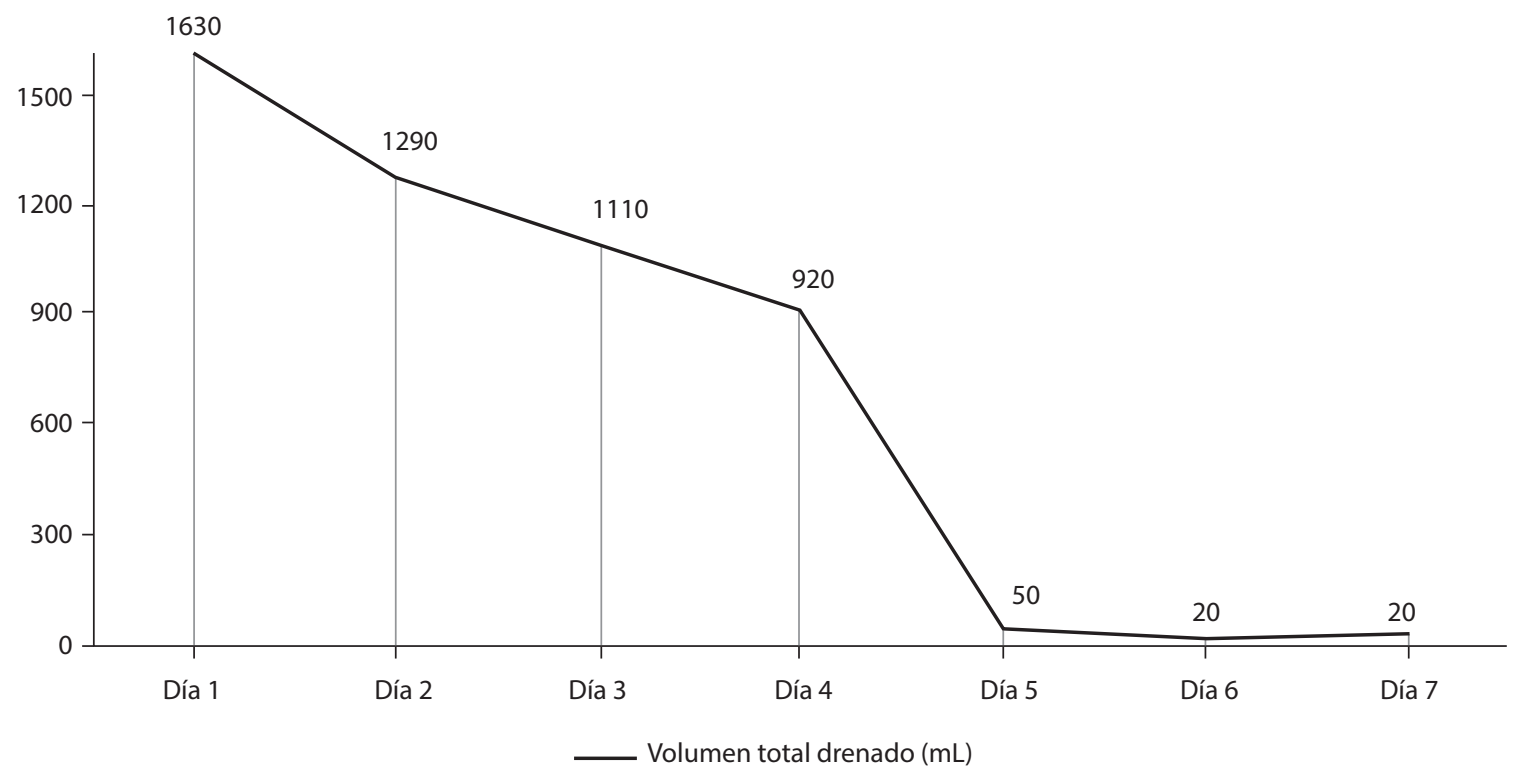

Figura 3. Disminución del volumen del drenaje pleural asociado a la Terapia Nutricional.

los reportes de caso en la literatura, la disminución del gasto quiloso es la manifestación de un tratamiento y

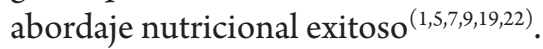

A pesar del impacto favorable de la intervención nutricional en la evolución del flujo quiloso desconocemos el impacto en el estado nutricional, ya que no se logró monitorear la composición corporal, la cual estaba prevista para el día que falleció. Se observaron solamente mejorías subjetivas en el edema en miembros inferiores, mejorías en la resequedad de la piel y menor pérdida de cabello, incremento en el apetito y una mejoría en la ventilación debido a la menor congestión pulmonar.

El estado nutricional de los pacientes con quilotórax se ve afectado ya que este se compone principalmente de grasas y proteínas. La pérdida de quilo y linfa conduce a una pérdida significativa de proteínas, inmunoglobulinas, grasas, vitaminas, electrolitos y agua ${ }^{(25,26)}$. Además, el drenaje elevado del mismo, con pérdida continua de linfocitos, conlleva al paciente a la inmunodeficiencia, a una mayor susceptibilidad a infecciones y aumento en la mortalidad ${ }^{(14)}$. Debido a la ausencia de información con respecto al abordaje nutricional, uno de los factores a tomar en cuenta es la evolución clínica del paciente, monitoreando estrechamente todos los puntos que integran la evaluación del estado nutricional, lo cual nos dará la pauta para hacer modificaciones en la terapia nutricional. La valoración clínica enfocada en nutrición aún se encuentra rezagada, y sabemos que cuando existen signos clínicos de deficiencia instaurados en el paciente, estas se abordan de manera tardía. Cabe recalcar que el quilotórax compromete el estado nutricional del paciente, por lo cual el abordaje debe ser multidisciplinario ${ }^{(27,28)}$.

En el caso reportado se evidencia que la rápida y especializada atención nutricional debe integrarse como parte fundamental del tratamiento, e instaurada por profesionales de la nutrición clínica, ya que en ciertos centros hospitalarios todavía existe desconocimiento sobre la importancia de las intervenciones nutricionales.

\section{CONCLUSIÓN}

La administración de terapia nutricional mixta (oral y NP) al paciente con diagnóstico de quilotórax secundario a bloqueo del conducto torácico por linfoadenopatía permitió la disminución del gasto quiloso, presentándose cambios en la composición y aspecto del mismo. Teniendo en cuenta la importancia del estado nutricional en la evolución de estos pacientes se propone un algoritmo basado en la evaluación nutricional que permita facilitar la toma de decisiones del profesional.

\section{Financiamiento}

El presente manuscrito fue elaborado sin el financiamiento de algún organismo público o privado. 


\section{Conflictos de intereses}

Los autores declaran no tener conflicto de intereses.

\section{Declaración de autoría}

Todos los autores participaron en la redacción del artículo, la concepción, la realización y el desarrollo del caso. Todos los autores revisaron el artículo y validaron su versión final.

\section{Referencias bibliográficas}

1. Sirvent Ochando M, López Villodre P, Martinez Seguí MJ. Soporte nutricional y tratamiento con octreotido del quilotórax. Nutr Hosp. 2010; 25(1): 113-9.

2. McGrath EE, Blades Z, Anderson PB. Chylothorax: aetiology, diagnosis and therapeutic options. Respir Med. 2010; 104(1):1-8.

3. Herrera-García JC, Sánchez-Pérez R. Derrame pleural: ruta diagnóstica inicial. Med Int Méx. 2015;31:181-190.

4. Froudarakis ME. Diagnostic Work-Up of Pleural Effusions Respiration. Respiration. 2008; 75(1):4-13.

5. Mechán V, Morón J, Salas A, Cevallos P, Llanos F, Rojas L, et al. Quilotórax bilateral asociado a linfoma no Hodgkin, folicular. Génesis del quilotórax. Acta Méd. Peruana. 2011; 28(2): 82-6.

6. Carrillo-Esper R, Sosa-García JO, Carrillo-Córdova CA. Quilotórax secundario a herida por proyectil de arma de fuego. Cir Cir. 2009;77(6):479-82.

7. García-Padilla E, Flores-López EN, Aguilar-de la Torre DL, Ronquillo-Pérez X. Tratamiento médico de quilotórax espontáneo. Med Int Méx. 2015; 31:110-2.

8. Machado JD, Suen VM, Marchini JS. Is oral nutritional therapy effective for the treatment of chylothorax? A case report. Nutrition. 2008;24(6):607-9.

9. Solís-Torres J, Méndez-Jiménez E. Quilotórax bilateral posterior a trauma de tórax cerrado. Acta Méd Costarric. 2014; 56(3):125-7.

10. Benítez I, Copons C, Castillo F. Tratamiento del quilotórax. An Pediatría Contin. 2008;6(3):159-65.

11. Sáyago-Ayerdi SG, Vaquero MP, Schultz-Moreira A, Bastida S, Sánchez-Muniz FJ. Utilidad y controversias del consumo de ácidos grasos de cadena media sobre el metabolismo lipoproteico y obesidad. Nutr Hosp. 2008; 23(3):191-202.

12. Bozzetti F, Arullani A, Baticci F, Terno G, Ammatuna M, Cappello G. Management of lymphatic fistulas by total parenteral nutrition. JPEN J Parenter Enteral Nutr. 1982; 6(6):526-7.

13. Nadolski G. Nontraumatic Chylothorax: Diagnostic Algorithm and Treatment Options. Tech Vasc Interv Radiol. 2016;19(4): 286-90.
14. Villabos-Mora C. Mora-Hernández GA. Quilotórax. Rev Med Cos Cen. 2015; 72 (615):385-9.

15. Nussenbaum B, Liu JH, Sinard RJ. Systematic management of chyle fistula: the Southwestern experience and review of the literature. Otolaryngol Head Neck Surg. 2000;122(1):31-8.

16. López MV, Luengo LM, Rangel JF, Liso FJ. Eficacia y seguridad del octreótide asociado a nutrición parenteral total en el tratamiento conservador del quilotórax. Nut Hosp. 2012;27(3):948-9.

17. Sanchez Alvarez C, Berenguel Espinosa JL, Aranaga FML. Fallo gastrointestinal agudo en el paciente crítico. Nutr Clin Med. 2017;11(2):59-73.

18. Singer P, Blaser AR, Berger MM, Alhazzani W, Calder PC, Casaer MP, et al. ESPEN guideline on clinical nutrition in the intensive care unit. Clin Nutr. 2018; 38(1):48-79.

19. García J, Alemán C, Jáuregui A, Vázquez A, Persiva O, Fernandez de Sevilla T. Quilotórax en adultos. Revisión de la literatura a partir de una serie de 17 casos. Arch Bronconeumol. 2017; 53(7):407-8.

20. Lesina BB, Aburto BM. Uso de octreotide (análogo de somatostatina) en un caso de quilotórax bilateral secundario a linfoma. Rev. Méd. Chile. 2005; 133: 1473-6.

21. Ramos W, Faintuch J. Nutritional Management of Thoracic Duct Fistulas. A Comparative Study of Parenteral Versus Enteral Nutrition. JPEN J Parenter Enteral Nutr. 1986;10(5): 519-21.

22. Gutiérrez Macias A, Lizarralde Palacios E, Merino Múgica JM, Cabeza García S, Martínez Odriozola P, De la Villa FM. Quilotórax bilateral en un caso de adenocarcinoma metastásico de primario desconocido. Ana Med Interna (Madrid). 2006;23(4):176-8.

23. Schurink B, Mazza E, Ruurda JP, Roeling TAP, Steenhagen E, Bleys RLAW, et al. Low fat tube feeding after esophagectomy is associated with a lower incidence of chylothorax. Ann Thorac Surg. 2019: pii:S0003-4975(19)30412-6.

24. Zasada IA, Banner J, Bugge A. "Nutrition or no nutrition?": Chylothorax or leakage of total parenteral nutrition? Forensic Sci Med Pathol. 2019.

25. Nair SK, Petko M, Hayward M. Aetiology and management of chylothorax in adults. Eur J Cardiothorac Surg. 2007;32(2):362-9.

26. Mallick A, Bodenham AR. Disorders of the lymph circulation: their relevance to anaesthesia and intensive care. Br J Anaesth. 2003;91(2):265-72.

27. Palmeiro R, Arosa V, Cuerda C, Bretón I, Camblor M, Rodríguez $\mathrm{MC}$, et al. Quilotórax persistente en paciente con linfangioleiomiomatosis. Nutr. Hosp. 2012; 27(3):940-2.

28. Cortés-Télles A, Rojas-Serrano J, Torre-Bouscoulet L. Quilotórax: frecuencia, causas y desenlaces. Neumol Cir Torax. 2010; 69(3):157-62. 